\title{
Environmental Health Insights
}

OPEN ACCESS

Full open access to this and thousands of other papers at http://www.la-press.com.

\section{Environmental Health Resilience}

\author{
Tim Kelley
}

Environmental Health Sciences Program, Department of Health Education and Promotion, College of Health and Human Performance, East Carolina University, Greenville, North Carolina, United States.

Corresponding author email: kelleyt@ecu.edu

\section{Global Human Populations}

The capacity of the Earth's environment to support increasing and expanding human populations has been questioned at least for hundreds of years, but never more than in the mid to late 20th Century and early 21st Century. Global human population now exceeds seven billion and continues to increase at an unprecedented rate. Estimates of future (2050) human populations on Earth range from a low of about 7.4 billion to a high of 10.6 billion ("United Nations World Population to 2300", 2004 accessed at http://www.un.org/esa/population/publications/ longrange2/WorldPop2300final.pdf). Current human populations already place an extreme burden on global environmental resources, including air, water and food quality as well as increasing challenges related to human waste management and disease prevention, control and treatment. In fact, some have proposed that humans have entered the "anthropocene", an age in which the global environment is dominated by human activities (http:// www.sciencedaily.com/releases/2012/11/121101131609.htm).

Climate change and expanding human populations contribute to increased risk of transmission of infectious and non-infectious disease. Developing nations with huge human populations such as China and India are benefitting from increased economic globalization, allowing for increased availability of personal luxuries such as automobiles, which in turn results in increased pollution and further depletion of natural resources such as global oil reserves. Increasing availability to global resources also may contribute to global conflict over environmental resources such as oil, water and food. In the United States, 2013 was the hottest year on record. Average global temperatures are also on the rise, with Australia being another prime example. Globally, 2012 was the tenth hottest year on record since data collection began in 1880 (http:/www.ncdc.noaa.gov/sotc/global/2012/13).

Many people are now starting to question the ability of human populations to continue to grow, and perhaps even for humans continue to exist on the planet without significant changes in the way that we interact with our global environment. Others point out that dire predictions of the fragility of humanity have been made for thousands of years and that humans have continued to survive and even grow in spite of these challenges.

Environmental Health Insights 2013:7 29-31

doi: $10.4137 /$ EHI.S11687

This article is available from http://www.la-press.com.

() the author(s), publisher and licensee Libertas Academica Ltd.

This is an open access article. Unrestricted non-commercial use is permitted provided the original work is properly cited. 


\section{Environmental Terminology: Sustainability vs. Resiliency}

Terminology used in environmental science and policy tends to evolve over time.

One term that has been used in the past to describe humans ability to utilize resources within a range that can be maintained to support humans and other environmental systems for the long-term, is sustainability. However, this term has been perceived by some as potentially limiting human economic growth, and conversely by others as precluding the possibility of improvement (eg, by appearing to promote maintenance of the current state of the environment). The term renewable also relates to sustainability, since renewable resources are capable of replacing themselves, given the opportunity, and perhaps some human encouragement to do so (forests may serve as an example). Conversely, non-renewable resources are not capable of replacing themselves within reasonable human time frames (eg, petroleum and "fossil" water aquifers). Agricultural crops and water resources may be classified into a category of "semirenewable", since they may be renewed within reasonable human time frames with the help and support of human ingenuity and technology. Since significant technological advances have occurred during the past 100 years, humans have already extended resources well beyond the "worst-case-scenario" that supplies of resources such as food, water and petroleum would be exhausted as early as the year 2000. Humans have demonstrated the ability to cope with changing environments for thousands of years. The question then becomes, can humans maintain this ability to cope with environmental changes indefinitely, and if not, at what point will the consequences of our inability to cope result in significant consequences for humans and our environment?

Another term that has been applied to the environment more recently as a supplement or alternative to sustainability is resilience. Resilience has been proposed by some as the new "buzzword" or "lingo" of environmental science and ecology, replacing or augmenting use of the term sustainability. When this definition is applied to our environment (as in the study of ecology), ecological resilience may be defined as: "the capacity of an ecosystem or natural population to resist or recover from major changes in structure and function following natural or human-caused disturbances, without undergoing a shift to a vastly different regime that is undesirable and very difficult to reverse from a human perspective" (http://www. mpa.gov/resources/glossary/). Therefore, natural and even human-constructed (anthropogenic) systems have a tendency to return to a natural "steady-state" condition and can return to this previous condition following changes. Some systems can return very close to their former conditions following significant and even repeated changes (the example of stretching a rubber band), exhibiting a high level of resilience. Others, once changed, can never return to their former state, or require significant energy input to return to their former state (a straight metal wire, once bent beyond a certain point, may never be completely straight again, or require significant input of energy to straighten it). There may also be changes to both resilient and non-resilient systems that are not obvious (repeatedly stretching a rubber band or bending a metal wire weakens the material so that it is more susceptible to breakage than before the deformations began). Obviously, environmental systems are much more complex than the example of a rubber band or a metal wire, and may have the capacity to "heal" themselves, and even, in some cases, become stronger after the damage than before (for example, a broken bone, once properly mended, may be stronger than before the break).

\section{Environmental Health Resilience}

How could this concept of resilience be applied to the discipline of environmental health, for example, in the area of water quality? The global hydrological cycle both contaminates and then purifies water as it evaporates, condenses, precipitates, infiltrates and percolates through natural atmospheric and terrestrial systems. This cycle is almost entirely powered by the Sun, and very little water is lost to space from the Earth's atmosphere, so this would seem to be a highly sustainable and therefore resilient system. However, the speed and volume of water use has increased so much due to increasing human demands that the amount of time for water to be purified by this natural system is not adequate to meet localized human demands. Anthropogenic technology-based drinking water and wastewater purification systems mimic the 
natural hydrologic cycle in many ways, but require significantly increased energy input in the form of petroleum and human resources. The vulnerability of water treatment facilities to natural and human-made disasters substantially reduces the resilience of these systems. Given an additional input of energy following disasters, these systems may recover, but perhaps not quickly enough to prevent human suffering and environmental damage.

Food availability and quality is another example in which the natural system provides for resilience (reproduction of animal and plant species), so long as the demand is not too great. As prehistoric human "hunter-gatherer" societies were replaced with agrarian societies, human population increased exponentially due to increased food availability and improved food storage techniques. However, human populations are now increasingly dependent upon "monoculture" agricultural crops, which are more susceptible to both natural and human-made challenges (eg, disease and climate change). Again, this is an example of increased human population density reducing the natural resilience of the system. Another way of thinking about this is that the natural "buffer" of resilience has been decreased through increasing human demands on limited environmental resources. Whereas in the past, small human populations might have withstood significant changes in our environment due to human adaptability and flexibility, our modern lifestyle has restricted our range of acceptable conditions as well as our tolerance for change. While small, isolated groups of humans might be able to ultimately survive shortterm catastrophes, long-term human survival following a global catastrophe is not guaranteed, and perhaps not even likely. A key component of human system (including environmental health) resilience is increased planning for wider ranges of both natural and anthropogenic changes (eg, climate, sea-level, terrorism) in order to more quickly and appropriately respond to these changes. This environmental "hazard mitigation" or "planned adaptation" is presented here as planning for potential non-historically-predictable future environmental health challenges vs. "emergency response" to more random, sporadic, relatively short-lived challenges.

\section{Conclusion}

Clearly, humans have proven to be highly adaptable to both environmental and social changes. This adaptability is one of the primary traits that have allowed humans to become the dominant species on Earth. We have been able to overcome or avoid many of the "checks and balances" normally imposed upon the growth of natural systems such as varying food and water supplies, shelter, and disease that have limited other species from achieving global domination. However, our dominance comes with a price - that of being more vulnerable to natural and anthropogenic changes that could ultimately replace us as a dominant species. We should therefore consider ways in which we can increase resilience as much as possible within both natural systems, human-made technological systems and environmental health systems so that we can better respond to and recover from challenges from our environment and ourselves.

\section{Author Contributions}

Wrote the first draft of the manuscript: TK. Made critical revisions and approved final version: TK. The author reviewed and approved of the final manuscript.

\section{Funding}

Author(s) disclose no funding sources.

\section{Competing Interests}

Author(s) disclose no potential conflicts of interest.

\section{Disclosures and Ethics}

As a requirement of publication the author has provided signed confirmation of compliance with ethical and legal obligations including but not limited to compliance with ICMJE authorship and competing interests guidelines, that the article is neither under consideration for publication nor published elsewhere, of their compliance with legal and ethical guidelines concerning human and animal research participants (if applicable), and that permission has been obtained for reproduction of any copyrighted material. This article was subject to blind, independent, expert peer review. The reviewers reported no competing interests. 